https://helda.helsinki.fi

Evolution of migration rate in a spatially realistic metapopulation model.

\author{
Heino, Mikko
}

Chicago, USA: University of Chicago Press.

2001

American-Naturalist. 2001; 157(5): 495-511

http://hdl.handle.net/1975/5157

http://dx.doi.org/doi:10.1086/319927

Downloaded from Helda, University of Helsinki institutional repository.

This is an electronic reprint of the original article.

This reprint may differ from the original in pagination and typographic detail.

Please cite the original version. 


\section{Evolution of Migration Rate in a Spatially Realistic Metapopulation Model}

\author{
Mikko Heino ${ }^{1,2, *}$ and Ilkka Hanski ${ }^{3, \dagger}$ \\ 1. Division of Population Biology, Department of Ecology and \\ Systematics, University of Helsinki, Box 17, FIN-00014 Helsinki, \\ Finland; \\ 2. Adaptive Dynamics Network, International Institute for Applied \\ Systems Analysis, A-2361 Laxenburg, Austria; \\ 3. Metapopulation Research Group, Division of Population \\ Biology, Department of Ecology and Systematics, University of \\ Helsinki, Box 17, FIN-00014 Helsinki, Finland
}

Submitted January 3, 2000; Accepted December 26, 2000

ABSTRACT: We use an individual-based, spatially realistic metapopulation model to study the evolution of migration rate. We first explore the consequences of habitat change in hypothetical patch networks on a regular lattice. If the primary consequence of habitat change is an increase in local extinction risk as a result of decreased local population sizes, migration rate increases. A nonmonotonic response, with migration rate decreasing at high extinction rate, was obtained only by assuming very frequent catastrophes. If the quality of the matrix habitat deteriorates, leading to increased mortality during migration, the evolutionary response is more complex. As long as habitat patch occupancy does not decrease markedly with increased migration mortality, reduced migration rate evolves. However, once mortality becomes so high that empty patches remain uncolonized for a long time, evolution tends to increase migration rate, which may lead to an "evolutionary rescue" in a fragmented landscape. Kin competition has a quantitative effect on the evolution of migration rate in our model, but these patterns in the evolution of migration rate appear to be primarily caused by spatiotemporal variation in fitness and mortality during migration. We apply the model to real habitat patch networks occupied by two checkerspot butterfly (Melitaea) species, for which sufficient data are available to estimate rigorously most of the model parameters. The modelpredicted migration rate is not significantly different from the empirically observed one. Regional variation in patch areas and connectivities leads to regional variation in the optimal migration rate, predictions that can be tested empirically.

\footnotetext{
* Corresponding author; e-mail: mikko.heino@helsinki.fi.

${ }^{\dagger}$ E-mail: ilkka.hanski@helsinki.fi.
}

Am. Nat. 2001. Vol. 157, pp. 495-511. (c) 2001 by The University of Chicago. 0003-0147/2001/15705-0003\$03.00. All rights reserved.
Keywords: checkerspot butterflies, individual-based model, metapopulation, migration propensity.

Evolution of migration rate is one of the better studied topics in evolutionary ecology (Gadgil 1971; Comins et al. 1980; Olivieri and Gouyon 1997), inspired by the wide range of migration rates and patterns exhibited by animals and plants. A large number of explanations based on both group and individual selection arguments for more or less migration, which may be unconditional or conditional on the state of the individual or its environment, has been put forward in the literature (reviewed by Johnson and Gaines 1990; McPeek and Holt 1992; Gandon and Michalakis 2001; Ronce et al. 2001).

Recently, the selection that increasing loss and fragmentation of natural habitats may impose on the evolution of migration rate has received much attention (Leimar and Norberg 1997; Travis and Dytham 1998, 1999). Given the various costs and benefits of migration and the various types of habitats (ephemeral or stable), it is not obvious which way habitat fragmentation might select migration rate to evolve. According to one view, high migration rate leads to elevated mortality, and as migration mortality is likely to increase with increasing habitat fragmentation, a lower migration rate is expected to evolve. According to an opposing view, migration is likely to increase when the environment becomes increasingly fragmented, extinction rate increases, and an increasing fraction of suitable habitat is unoccupied and available for colonization. In this situation, risk of mortality during migration may be more than balanced by a chance of finding an uncrowded patch with little or no competition, and high migration rate may evolve even if mortality during migration might be high (Comins et al. 1980). At the same time, increased migration can be either beneficial or detrimental for metapopulation survival.

These two viewpoints relate to two extreme situations that are well documented for natural populations. If there is just a single isolated population living in a stable habitat, selection is expected to operate against emigration from that population. Accordingly, populations on isolated oce- 
anic islands and mountaintops tend to show low migration rate and capacity (Hesse et al. 1951; Roff 1990). In the other extreme, only species with high migration rates may survive in ephemeral habitat patches with fast turnover. Rare species that occur in naturally patchy or fragmented habitats have indeed high migration rates (Brown 1951; Southwood 1962; Roff 1994).

With human-caused habitat fragmentation, the situation is less clear-cut, and an appropriate model is required to take into account the relevant biology of the species and the key features of landscape structure. Studies of the evolution of migration rate have been mostly based on general models that necessarily make many simplifying assumptions. Though such models have definite advantages, they also have the drawback that producing testable quantitative predictions is difficult or impossible. It may also be difficult to ascertain to what extent the results are sensitive to the simplifying structural assumptions made in the model. Thus, some general models of the evolution of migration assume an infinite number of habitat patches, no explicit spatial structure in the landscape, and recolonization that occurs immediately following population extinction (e.g., Gandon and Michalakis 1999; Ronce et al. 2000). In this case, colonization is effectively decoupled from migration, and the model should be used cautiously to draw inferences about the consequences of habitat fragmentation on the evolution of migration rate. In other models, migration is modeled on a regular lattice, with random movements between the neighboring lattice cells (Travis and Dytham 1998, 1999). Such models implicitly assume that migrating individuals have no power to search actively for suitable habitat, unlike the myriads of animal species that inhabit naturally patchy habitats.

In this article, we have adopted a more specific modeling approach, complementing an expanding body of empirical literature. Many recent metapopulation studies have documented the dynamics of species living in highly fragmented landscapes, which can be described as irregular networks of patches of unequal size (Hanski 1998, 1999). Much of this work has been done on butterflies (Thomas and Hanski 1997), which is also the focal taxon in our study. In particular, we take advantage of two empirically based models to parameterize a submodel for the movements of individuals among habitat patches and to parameterize another submodel for long-term metapopulation dynamics. To these models, we add an individual-based simulation to model the evolution of migration rate. Individual-based models are often plagued by many untested assumptions and parameters, but this is not the case here because of the wealth of information available for the focal species, making it possible to parameterize rigorously the two submodels with empirical data. To some extent, our model can also be used to test the robustness of the predictions of previous simpler models.

We start by describing the model and how it has been parameterized for the butterfly metapopulations. Comparable models can be constructed for other species with different spatial population structure and different life histories by modifying relevant model parameters and assumptions. The "Results" section is divided into two parts. The purpose of the first part is to study more general questions about the evolution of migration rate in response to habitat loss and fragmentation by assuming a regular lattice structure for the landscape. These results also allow us to compare the predictions of our model with the predictions of other models. In the second part, we demonstrate how the model can be applied to real metapopulations living in real fragmented landscapes to generate testable quantitative predictions.

\section{Individual-Based Model}

In this section, we describe the evolutionary model and its components. The backbone of the model is a description of the life cycle of the focal species. If the model were to be applied to other species than the butterflies considered here, the first task would be to make the necessary modifications in the description of the life cycle in the simulation model. Being a model of the evolution of migration rate, a key component of the model is a submodel of the movement behavior of individuals (adult individuals in the case of butterflies). For this purpose, we use a previously described statistical model that can be parameterized with empirical mark-release-recapture data. This submodel covers only the migration period in one generation, whereas in the evolutionary model, we have to model longterm dynamics. To ensure that our model predicts realistic long-term dynamics, we tune some parameter values such that the individual-based evolutionary model produces similar year-to-year dynamics than a previously parameterized ecological metapopulation model. We thus require the evolutionary model to exhibit ecologically realistic behavior. Having thus constructed the model, we allow one parameter, the propensity to migrate, to evolve, while other parameters are fixed to the values estimated from empirical data. We now turn to a description of the model components and the way it was parameterized for the focal taxa in this study.

\section{Life-History Model}

The model assumes the following life cycle, which corresponds closely with the life cycle of the Glanville fritillary butterfly (Melitaea cinxia) and related checkerspot butterflies (Kuussaari 1998; Hanski 1999). These species are 
the focal taxa to which the model will be applied in "Results." Most of the model parameters can be estimated with data available for M. cinxia, but the best data on adult movements come from the related species Melitaea diamina, which is ecologically very similar to M. cinxia (Wahlberg et al. 1996). All the parameters are summarized in table 1 .

We assume a highly fragmented landscape, consisting of discrete patches of suitable habitat surrounded by uniformly unsuitable habitat. There is one generation per year, and the adults, the dispersive stage in the life cycle, hatch in early summer. Females mate only once, and after mating, they start to search for host plants suitable for oviposition. The search continues until they die or the season ends, after day 30; very few females survive until the end of the season. During the search, females may migrate to another habitat patch. Eggs are laid in large batches, at most one egg batch per day. Each egg batch develops into one larval group. The larvae remain in sib groups until the next spring, and thus larval group is the demographic unit for most of the year. Survival of the larval groups until autumn is density dependent because the availability of suitable host plants is limited. We assume that local carrying capacity sets an upper limit to the number of surviving larval groups. If population size is greater than the carrying capacity, randomly selected larval groups are removed until the carrying capacity is reached. Below the carrying capacity, there are no adverse effects of density dependence. Mortality during winter and spring is density independent.

\section{Migration of Adults}

Migration is modeled using the virtual migration model of Hanski et al. (2000). This model consists of a set of structural assumptions about the influence of habitat patch area and isolation on survival and migration (the biological model) and a statistical model for maximum-likelihood parameter estimation from mark-release-recapture data. The model assumes identical and independent individuals, and essentially the purpose is to model the individual capture histories as recorded in a field study. We use the virtual migration model as a well-parameterized submodel for migration in our individual-based model of the evolution of migration rate.

Here we focus on describing the biological model; the original paper (Hanski et al. 2000) should be consulted for further details and on the method of parameter esti-

Table 1: Model parameters and their values for checkerspot butterflies

\begin{tabular}{|c|c|}
\hline Parameters & Value \\
\hline \multicolumn{2}{|l|}{ Larval groups: } \\
\hline Area-dependent carrying capacity $\left(K_{i}\right)$ & $10 A_{i}^{0.5 \mathrm{a}}$ \\
\hline Mean diapause survival of larval groups $\left(s_{w}\right)$ & $.4^{\mathrm{b}}$ \\
\hline Between-patch variance in diapause survival $\left(\sigma_{s_{w}}^{2}\right)$ & $.0092^{\mathrm{c}}$ \\
\hline Correlation in diapause survival $\left(\rho_{s_{w}}\right)$ & $.25^{\mathrm{c}}$ \\
\hline Mean larval group size $(f)$ & $12^{\mathrm{b}}$ \\
\hline$k$ of the negative binomial distribution for larval group size & $3^{c}$ \\
\hline Within-patch correlation in larval group size $\left(\rho_{f}\right)$ & $.75^{\mathrm{c}}$ \\
\hline \multicolumn{2}{|l|}{ The virtual migration model: ${ }^{\mathrm{d}}$} \\
\hline Within-patch survival probability $(\phi)$ & $.890(.868-.935)$ \\
\hline Migration propensity $(\eta)$ & $.130(.103-.176)$ \\
\hline Scaling of emigration $\left(\zeta_{\mathrm{em}}\right)$ & $.170(.000-.414)$ \\
\hline Scaling of immigration $\left(\zeta_{\mathrm{im}}\right)$ & $.263(.000-.577)$ \\
\hline Distance dependence $(\alpha)$ & $4.910(3.798-6.138)$ \\
\hline Migration mortality $(\lambda)$ & $.175(.061-.517)$ \\
\hline \multicolumn{2}{|l|}{ Adults: } \\
\hline Daily probability of laying an egg batch & $.33^{\mathrm{e}}$ \\
\hline \multicolumn{2}{|l|}{ Evolution of migration rate: } \\
\hline Number of loci & 32 \\
\hline Mutation probability per locus & .001 \\
\hline 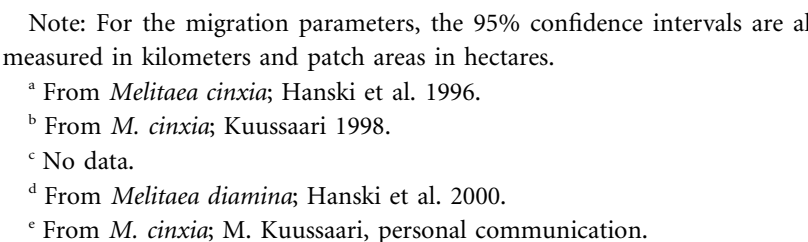 & available. Distances are \\
\hline
\end{tabular}


mation. The virtual migration model is based on discretized histories of individuals. In the course of unit time, which is $1 \mathrm{~d}$ in our application for butterflies, events are assumed to occur in the following order. First, individuals have a daily within-patch survival probability $\phi$ before possibly emigrating. Second, surviving individuals have a patch-specific probability of emigrating from patch $j$, which is a power function of patch area $A_{j}$ :

$$
\epsilon_{j}=\eta A_{j}^{-\xi_{\mathrm{em}}},
$$

where $\zeta_{\mathrm{em}}$ is a scaling constant and parameter $\eta$ is termed migration propensity. Third, the probability of surviving migration increases with increasing connectivity $S_{j}$ of patch $j$ and is given by

$$
S_{j}^{2} /\left(\lambda+S_{j}^{2}\right)
$$

where $\lambda$ is a parameter describing migration mortality: large values result in low survival. Connectivity of patch $j$ is measured by

$$
S_{j}=\sum_{k \neq j} \exp \left(-\alpha d_{j k}\right) A_{k}^{\zeta_{k}},
$$

where $d_{j k}$ is the distance between patches $j$ and $k, \alpha$ scales distance dependence of migration (if $\alpha \gg 0$, distant patches contribute very little to the connectivity of patch $j)$, and $\zeta_{\text {im }}$ scales patch area dependence of immigration. Probability of arriving at patch $k$, given that an individual has emigrated from patch $j$ and survived migration, depends on the contribution of patch $k$ to the connectivity of patch $j$ and is given by $\exp \left(-\alpha d_{j k}\right) A_{k}^{S_{\text {im }} /} / S_{j}$. Taking mortality into account, the probability of an individual leaving patch $j$ to reach patch $k$ is given by

$$
\psi_{j k}=\frac{\exp \left(-\alpha d_{j k}\right) A_{k}^{\zeta_{\mathrm{im}}}}{\lambda / S_{j}+S_{j}} .
$$

These probabilities constitute a matrix that is used in this model to redistribute migrating individuals. Note that the presence of other patches influences $\psi_{j k}\left(\right.$ via $\left.S_{j}\right)$, and hence the model includes "competition" among the target patches for migrating individuals.

\section{Long-term Metapopulation Dynamics}

For our purposes, it is essential that the individual-based simulation model produces realistic long-term dynamics. To achieve this, we used the incidence function model (IFM; Hanski 1994), which has been previously widely applied to butterfly metapopulations (Hanski et al. 1996; Wahlberg et al. 1996; Hanski 1999). The IFM is a stochastic patch occupancy model, which incorporates a finite number of patches and spatial variation in patch areas and connectivities. The latter two factors influence, respectively, the rates of population extinction and colonization (for a description of the model and its application, see Hanski 1994, 1999; ter Braak et al. 1998; Moilanen et al. 1988).

\section{Parameterization for Checkerspot Butterflies}

We assume that survival of larval groups in diapause over winter and early spring follows a bell-shaped beta distribution, with the mean survival $s_{w}=0.4$, as observed in an intensive study of M. cinxia (Kuussaari 1998), and variance $\sigma_{s_{w}}^{2}$. To account for regional stochasticity (Hanski 1999), we assume that there is correlation $\rho_{s_{w}}$ in the survival among populations.

In the spring, shortly before the adults emerge, the average larval group size $f$ in M. cinxia is 12 larvae per larval group (Kuussaari 1998). We assume that larval group size follows negative binomial distribution with parameter $k$. Within-patch correlation in larval group sizes, $\rho_{f}$, reflects common factors influencing larval survival, for instance, risk of parasitism (Lei and Hanski 1998) and quality of host plants in the habitat patch (Hanski 1999).

Parameter values for the migration submodel have been estimated for M. diamina (table 1; Hanski et al. 2000). During their lifetime, female butterflies are assumed to lay one clutch of eggs with probability 0.33 in each day. This value takes into account that females do not lay at the maximal rate (one clutch per day) unless the environmental conditions are optimal (which they typically are not).

There are four parameters for which empirical estimates are currently unavailable: variance in the survival of larval groups during diapause $\left(\sigma_{s_{w}}^{2}\right)$, between-patch correlation in the survival in diapause and in spring before hatching $\left(\rho_{s_{w}}\right)$, parameter $k$ of the negative binomial distribution describing the sizes of larval groups, and within-patch correlation in the sizes of larval groups $\left(\rho_{f}\right)$. The values of these parameters were chosen within feasible limits in such a manner that the long-term dynamics of the individualbased model were similar to the dynamics predicted by the IFM. The tuning of these free parameters was done by simulating the dynamics of the individual-based model in the real network of 94 habitat patches inhabited by $M$. diamina in the Tampere region in Finland (Wahlberg et al. 1996). This is the same metapopulation from which the parameter estimates for the migration submodel were obtained. The carrying capacity is assumed to depend on patch area (in ha) according to the power function $K_{i}=10 A_{i}^{0.5}$, where the power is obtained from Hanski et al. (1996) and the coefficient was chosen to yield realistic 
densities. Simulations were initiated with the observed pattern of patch occupancy.

In selecting the values for the four free parameters, three criteria were used. First, the long-term average patch occupancy should be close to the observed value. Second, extinction risk should scale with patch area as predicted by the IFM. Third, the numbers of extinction and colonization events should be similar to the prediction of the IFM. The following parameterization gave the best fit among a large number of different combinations: $\sigma_{s_{w}}^{2}=$ $0.0092, \rho_{s_{w}}=0.25, k=3$, and $\rho_{f}=0.75$. These values are consistent with the known biology of the species. With this parameterization, the mean patch occupancy predicted by the individual-based model is very close to the value observed in the field and predicted by the IFM (table 2). Extinction risk in small patches is somewhat higher in the individual-based model than in the IFM, which results in higher turn-over rate in the former (table 2). The dynamics of this metapopulation are analyzed in "Results."

\section{Evolutionary Dynamics}

We assume that migration propensity $\eta$ (eq. [1]) depends on the genotype of an individual and that inheritance is clonal (haploid). No information exists on the actual genetic architecture of this trait. Because there is no indication of dispersal dimorphism in checkerspot butterflies, we assume that migration propensity is a quantitative trait influenced by many genes of small additive effects. We implement this assumption by representing genotypes as bit strings of 32 bits in length. Each bit corresponds to one locus, with possible alleles " 0 " and "1." Phenotype is given as the sum of 1's in the string, scaled in such a manner that the possible phenotypic values fall within a desired range. In our trial simulations, migration propensity did not evolve to values higher than $\eta \approx 0.2$, and hence we scale the range of values phenotypes can take between 0 and 0.25 . This gives a step size of $\sim 0.008$. Mutations occur with constant probability 0.001 independently in each locus. Thus, mutations have small phenotypic efects, and mutational steps are small. Note that one phenotype may be coded by many genotypes.

Measure of relatedness within populations relative to the average relatedness in the metapopulation as a whole is calculated as $r=\left(p_{p}-p_{m}\right) /\left(1-p_{m}\right)$, where $p_{p}$ is the average similarity of two adult individuals chosen from the same population and $p_{m}$ is the average similarity of two individuals chosen from the entire metapopulation (Pamilo 1984; Queller and Goodnight 1989). Similarity is measured as the proportion of loci with shared alleles. Only polymorphic loci were used in the calculations. This measure of relatedness is used to assess changes in relatedness when the values of model parameters are changed. However, because inheritance is haploid in the model but diploid in reality, the model-based relatedness estimates are likely to be higher than those observed in real butterfly metapopulations.

\section{Results}

\section{Migration Propensity and Dynamics in Simple Landscapes}

We first examine the dependence of population dynamics on the model parameters. To avoid possible complications arising from complex spatial structures of real metapopulations and to facilitate comparisons with previous models, we use a hypothetical landscape of 100 identical patches on a $10 \times 10$-square lattice. The distance between adjacent patches is $500 \mathrm{~m}$, and each patch has a carrying capacity, $K=5$, and an area, $A=1$. The daily emigration probability thus equals migration propensity (eq. [1]). Using the parameter values for the checkerspot butterflies as described in the previous section (table 1), this landscape is well connected and supports a viable metapopulation. The results are based on simulations of 1,100 generations, with the first 100 generations omitted to eliminate transient dynamics. Simulations were initialized with all patches occupied unless otherwise stated.

The influence of migration propensity on the dynamics is summarized in figure 1. A very low level of migration

Table 2: Comparison of the dynamical properties of the incidence function model (IFM) and the individual-based model (this study) for Melitaea diamina

\begin{tabular}{llccl}
\hline Model & Occupancy & $x$ & \multicolumn{1}{c}{$e$} & Turn-overs/yr \\
\hline Incidence function model & $.36(.05)$ & $.884(.257)$ & .014 & $3.7(.6)$ \\
Individual-based model & $.36(.04)$ & $.973(.050)$ & $.018(.003)$ & $8.6(1.3)$ \\
\hline
\end{tabular}

Note: The observed occupancy (fraction of occupied patches) in 1995 was $0.37 ; x$ and $e$ are the parameters of the extinction risk-patch area relationship, $E_{i}=e A_{i}^{-x}$. The values for the IFM are from Wahlberg et al. (1996; the other IFM parameters had the values $\alpha=1.0$ and $y=3.62$ ). For the individual-based model, the values are based on 10 simulation runs of 1,100 generations initiated with the observed occupancy pattern and the first 100 generations omitted. The values in parentheses are standard deviations, except for $x$ in the IFM (standard error). 

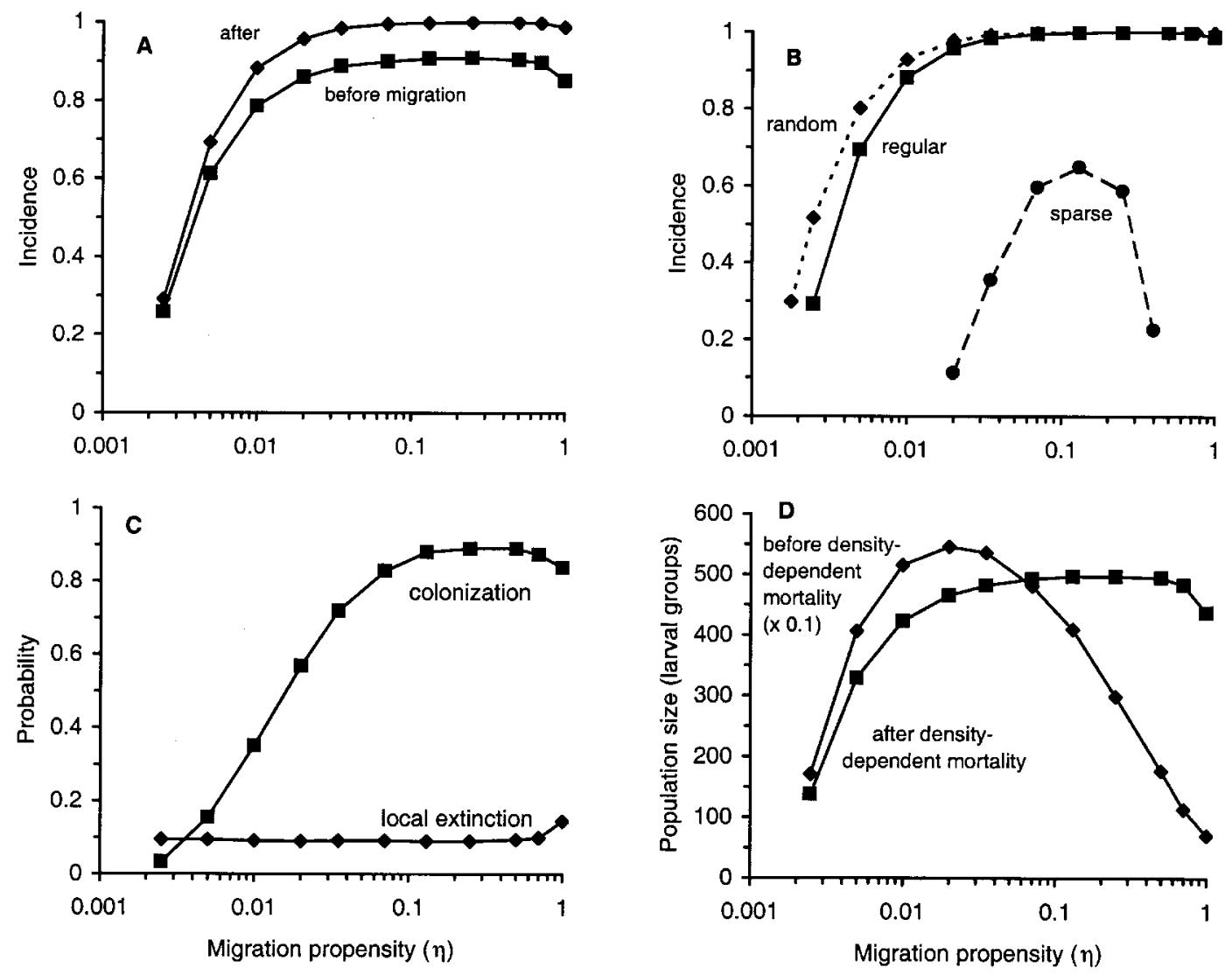

Figure 1: Dynamics and migration propensity in a hypothetical landscape (100 patches of unit area in a regular square lattice). Incidence is maximal after the migration phase in summer (upper line in $A$ ) and decreases thereafter to reach the minimum in the spring (lower line). Randomizing patch locations has no major influence on incidence, but increasing patch distances by $50 \%$ lowers incidence dramatically $(B)$. Local extinction risk is almost independent of migration rate, but colonization probability increases with increasing migration propensity $(C)$. Total metapopulation size after density dependence is primarily determined by incidence $(D)$. Parameter values are given in table 1 .

is sufficient to maintain a viable metapopulation: risk of metapopulation extinction is negligible for migration propensities $\eta>0.0025$. For higher levels of migration, incidence (the fraction of occupied patches) first increases rapidly, then reaches a plateau. For $\eta>0.02$, the incidence in the spring, before migration, is $\sim 0.9$, whereas following migration almost all patches are occupied (fig. 1A). There is thus no optimal migration propensity that would maximize the incidence. If individuals always migrate, incidence decreases slightly because of increased local extinction risk and the mortality during migration.

Colonization probability, which is here defined as the probability that a patch that is empty in the spring is occupied following the migration period, increases steadily with $\eta$ (fig. 1C). In contrast, local extinction risk, defined as the probability that a patch occupied in the spring goes extinct before the following spring, is hardly influenced at all by migration. There are two main reasons for the latter result. First, although roughly half of the emigrants die during migration, emigration is partly compensated for by immigration (the rescue effect). Second, even a fairly high emigration rate has no significant effect on the local population sizes after density dependence because fecundity is so high that local population sizes tend to be well above the carrying capacity before the operation of density dependence. For the same reason, total metapopulation size after density dependence is mainly determined by patch occupancy (fig. 1D). The total metapopulation size before density dependence is greatly influenced by losses during migration, and the metapopulation size peaks at a low migration propensity $(\eta \approx 0.02)$.

No real metapopulation exists on a regular square lattice. This assumption, however, is not critical for the predicted dynamics, which hardly change when the patch locations are randomized. The main differences are that, in a landscape with random patch locations, patch occupancy 
is slightly higher than with regular spacing of patches and that the metapopulation is viable for slightly lower migration propensity (fig. $1 B$, stippled line). This effect occurs because randomization of patch locations introduces some clustering of patches, increases average connectivity among patches, and thereby results in more efficient colonization of empty patches (Adler and Nuernberger 1994; Hanski and Ovaskainen 2000).

The dynamics are sensitive to changes in interpatch distances. We introduce another landscape by increasing the distances between the patches by $50 \%$ (increasing the value of the scaling parameter $\alpha$ would have exactly the same effect). This landscape will be referred to as "sparse." Dynamics in the sparse landscape are more sensitive to changes in migration propensity (fig. $1 B$, dashed line) than in the original "dense" landscape, and now a certain level of migration maximizes incidence. Colonization of empty patches is now more difficult than in the dense landscape because of high migration mortality (only $~ 5 \%$ of migrants survive). Indeed, too much migration leads to the extinction of the entire metapopulation (fig. $1 B$ ), as has been observed in general metapopulation models (Hanski and Zhang 1993).

\section{Evolution of Migration in Simple Landscapes}

Figure 2 illustrates the evolution of migration propensity in the landscape introduced in the previous section. The simulations were initiated with monomorphic populations with either high $(\eta=0.21$; fig. $2 A)$ or very low $(\eta=0.015$; fig. $2 B)$ migration propensity. In both cases, the sequence of events is similar. First, the population becomes polymorphic as mutations introduce new phenotypes. Strong directional selection favors phenotypes with intermediate migration propensities, and the population average changes. After about 500 generations, the metapopulation has evolved to the average migration propensity of $\sim 0.1$. Thereafter, both the average and the variance of the phenotypic distribution fluctuate, but there are no long-term trends: the metapopulation has attained a quasi-stationary phenotypic distribution. We term the long-term average of this distribution as the "evolutionarily optimal migration propensity." Note that this migration propensity is not optimal in the sense that it would maximize metapopulation size; rather, it is optimal in the sense that it is an approximation of an evolutionarily stable strategy. In all the subsequent simulations, optimal migration propensity and other statistics were calculated on the basis of at least five replicate simulations of 1,500 generations from which the first 500 generations were omitted.

We next study the influence of the model parameters on the evolutionarily optimal migration propensity. We first focus on distance dependence of migration, as de- termined by the parameter $\alpha$. Increasing $\alpha$ increases the effective distances between the patches and thereby leads to higher mortality and shorter average migration distances. The average migration distance is approximately given by $1 / \alpha$.

Distance dependence of migration has a strong influence on optimal migration propensity (fig. $3 A$ ). When distance dependence is weak (range, $\alpha=0-2$ ), migration propensity evolves to a relatively high level. Increasing distance dependence selects for decreased migration. However, this trend is ultimately reversed when distance dependence becomes strong ( $\alpha \approx 6$ or higher). For very strong distance dependence, the metapopulation is not viable.

At least three different selective forces seem to be responsible of the nonmonotonic dependence of optimal migration propensity on the parameter $\alpha$. The decline in migration propensity with increasing $\alpha$ (in the range $\alpha=2-6)$ is primarily driven by increasing migration mortality (fig. $3 B$ ). However, increasing distance dependence decreases connectivity in the metapopulation, and above certain levels, average relatedness begins to increase (fig. $3 C ; \alpha \approx 2$ ) and the incidence begins to decrease (fig. $3 B ; \alpha \approx 5$ ). These changes create a selection pressure for increased migration because of increasingly severe kin competition and because of higher payoffs for successful immigrants through weak or absent competition in the newly colonized patches. The relative strengths of the latter two selective forces will be examined in "Discussion."

A similar nonmonotonic pattern in optimal migration rate is obtained when migration mortality $(\lambda)$ is varied: migration propensity is high when the mortality cost of migration is very low, whereas when the cost is increased, migration propensity decreases (fig. 4A). However, for very high cost, there is again a marked increase in the optimal migration propensity. This pattern is associated with similar changes in incidence (fig. $4 B$ ) and relatedness (fig. 4C) as in the example involving $\alpha$ (fig. 3). A qualitatively similar result emerged when we made space implicit (by assuming that all interpatch distances are equal, $\alpha=0$ ) rather than explicit.

The sparse landscape incurs an additional mortality cost because of longer distances among the patches. In this case, the increasing relationship between migration mortality and the optimal migration propensity dominates the full range of $\lambda$ values. Close to the extinction threshold, the sparse landscape selects for substantially higher migration propensity than the dense one for the same value of $\lambda$ (fig. 4).

Migration propensity decreases monotonically with increasing local carrying capacity (fig. $5 A$ ). This decrease can be explained by two mechanisms. First, local extinction risk decreases with increasing carrying capacity; hence, there is a diminishing probability that an immigrant will 

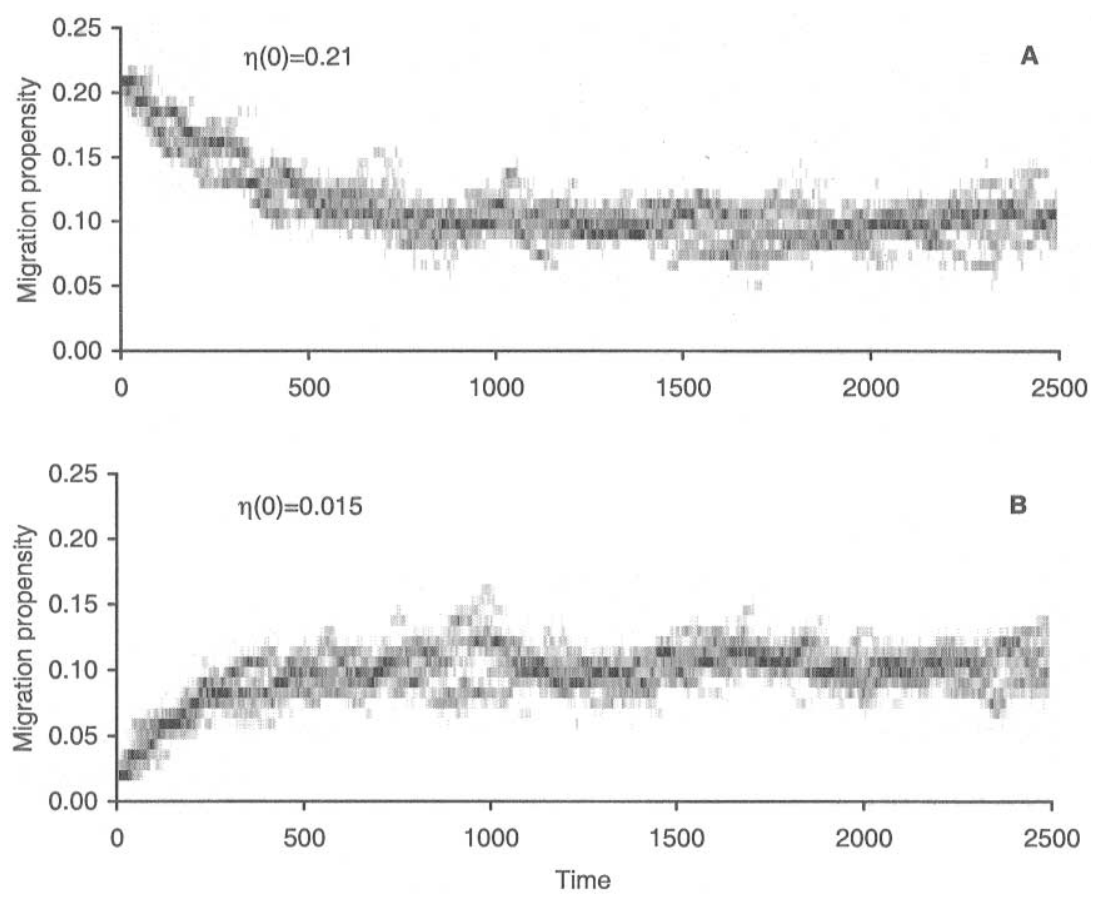

Figure 2: Evolution of the phenotypic distribution of migration propensity. After about 500 generations, the long-term mean of the distribution has converged to a constant value, regardless of the initial strategy $(A: \eta(0)=0.21 ; B: \eta(0)=0.015)$. Shading indicates the abundances of the phenotypes. Parameter values are given in table 1 .

arrive at an empty patch (fig. $5 B$ ), while mortality during migration is unaffected. Second, there is a monotonic decrease in relatedness with increasing carrying capacity (fig. $5 C)$.

The dependence of the evolutionarily optimal migration propensity on the carrying capacity is very similar in both the dense and sparse landscapes, in spite of much higher mortality cost of migration in the latter one. A likely explanation is that counteracting selective forces are roughly balanced: higher migration mortality selects for a decrease, whereas lower incidence and higher relatedness select for an increase in migration propensity.

Increasing average larval group size leads to only minor changes in local extinction risk and relatedness, and there are only marginal changes in the migration propensity. However, if larval groups are very small, local populations reach saturation less rapidly, and especially newly colonized patches become more prone to extinction. At the same time, relatedness increases. These changes favor an increase in migration propensity from $\eta \approx 0.103$ (when larval group size is 12 ) to $\eta \approx 0.124$ (when larval group size in the spring is three).

\section{Migration and Habitat Change}

The above examples can all be interpreted in terms of habitat change. Increase in distance dependence (fig. 3) or migration mortality (fig. 4) are likely to occur when the quality of the matrix habitat deteriorates. Destruction of some habitat patches would also increase migration mortality. The evolutionary response to such changes may be nonmonotonic, with migration propensity first decreasing, but eventually, when the extinction threshold is approached, there is an increase in migration propensity. Decrease in patch quality is likely to be manifested as a decrease in family size or in the carrying capacity (fig. 5). Such habitat changes would favor a monotonic increase in migration propensity.

A particular example of habitat quality deterioration is given in figure 6 . In this example, the original landscape is our dense landscape of 100 patches, each with a carrying capacity of $K=5$. We assume that during years $100-500$, carrying capacity in one patch is decreased by one unit in each year. By the year 500, all patches have reached unit carrying capacity (fig. 6A). If migration propensity is fixed to the value that is optimal in the original landscape $(\eta \approx 0.1)$, the metapopulation is not viable at time 500 (fig. $6 B$ ). However, if migration propensity is allowed to evolve, deteriorating habitat quality selects for increasing migration propensity, and the metapopulation is able to persist in the new landscape, although with a low incidence (fig. $6 C, 6 D$ ). In this example, evolution is able to rescue the metapopulation from extinction. Of course, whether 
such a rescue is likely to happen depends on the timescale of environmental change relative to the rate at which the metapopulation responds to selection.

\section{Dynamics and Evolution of Checkerspot Butterflies in Real Habitat Networks}

In this section, we move on to analyze the evolution of migration rate in real highly fragmented landscapes. A strength of this modeling approach is that it can be applied equally well to such irregular patch networks as to regular networks on lattice. We first focus on the patch network inhabited by Melitaea diamina in the Tampere region of Finland, consisting of 94 patches within an area 21 $\mathrm{km} \times 29 \mathrm{~km}$ (Wahlberg et al. 1996). Simulations were initialized with the observed pattern of patch occupancy (Wahlberg et al. 1996). The pattern of patch occupancy predicted by the model (fig. $7 \mathrm{~A}$ ) is very similar to the observed pattern (fig. 7B; from Wahlberg et al. 1996). Dense clusters of habitat patches support assemblages of relatively viable local populations, and the incidence is high in these clusters because of efficient recolonization. Isolated patches, if large enough, can remain occupied for a long period. However, once extinct, recolonization of isolated patches is unlikely and, hence, their incidences are low.

If migration propensity is allowed to evolve, keeping all the other parameters at their empirically estimated values, migration propensity decreases from the estimated value of $\eta=0.130$ to $\eta \approx 0.104$ (SD of 10 replicates $=0.005$ ). This "evolutionarily optimal" migration propensity is still within the 95\% confidence interval of the original parameter estimate (table 1). The pattern of patch occupancy remains practically unchanged, and there is no significant change in the overall level of patch occupancy. Migration propensity varies among patches from $\eta=0.09$ to $\eta=$ 0.13 (fig. $7 C$ ). Because of migration, patches close to each other tend to have similar values of $\eta$. There is a statistically nonsignificant tendency of migration propensity to be higher in patch clusters in which survival of migrants is high (fig. $7 D$ ). Average relatedness is very high $(0.955$, $\mathrm{SD}=0.008$ ) because of typically small local population sizes and clustering of patches.

Loss of habitat-be it total destruction of some patches or decrease in patch areas-has only minute evolutionary consequences on the migration propensity of M. diamina in the real landscape (table 3). In both cases, migration propensity tends to decrease, which is accompanied by a minute decrease in average patch occupancy. However, if only patch quality $(K)$ deteriorates, but patch areas stay the same, such that movement patterns between the patches remain unchanged, there is an increase in migration propensity (table 3 ).
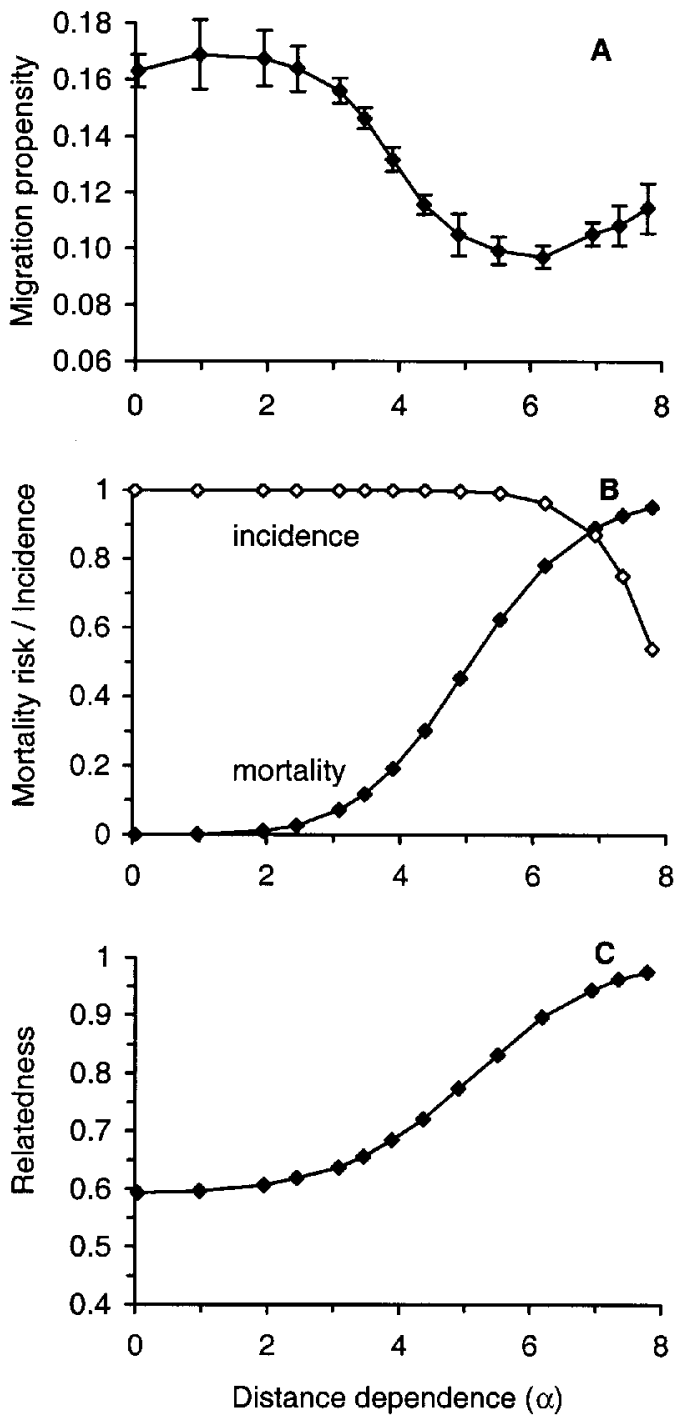

Figure 3: Evolutionarily optimal migration propensity has $\mathrm{U}$-shaped dependence on distance dependence of migration, parameter $\alpha(A)$. Vertical bars give the standard deviation in the results of six replicates. If distance dependence is low, isolation does not much hinder migration, and average migration distance is large. For strong distance dependence, migration is confined to the nearest neighbors, and for even larger values of $\alpha$, an extinction threshold is reached. Increasing distance dependence results in higher mortality during migration, which in turn decreases colonization rate and incidence $(B)$. Average relatedness increases with distance dependence $(C)$. Parameter values are given in table 1 .

We next use the model to study evolution of migration rate in the related species Melitaea cinxia in the Aland Islands in southwest Finland. The two Melitaea species share the same basic biology, to the extent that parameter values of the IFM estimated from M. cinxia (Hanski et al. 1996) predicted well the distribution of $M$. diamina in its 

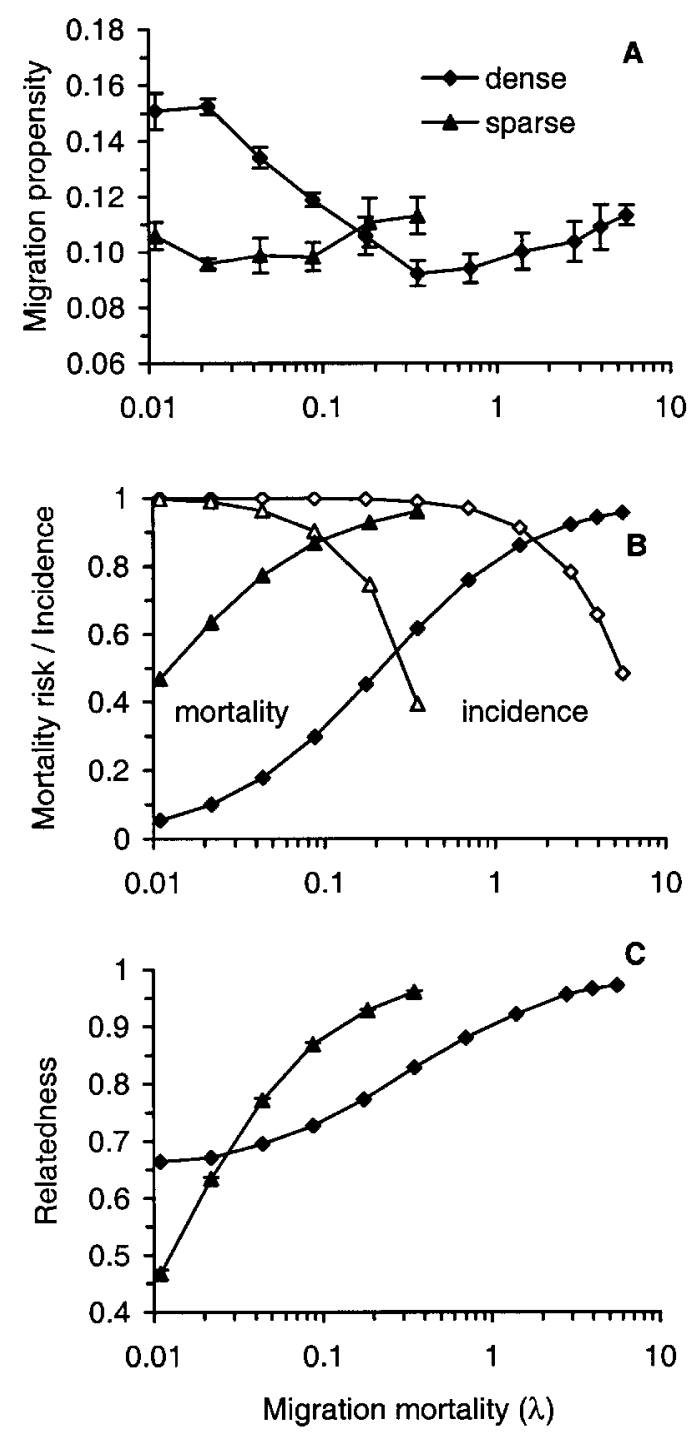

Figure 4: $A$, Evolutionarily optimal migration propensity in dense (diamonds) and sparse (distances increased by $50 \%$; boxes) networks for different values of migration mortality $(\lambda)$. When mortality is very high, incidence declines to a low level $(B)$. Metapopulation extinction will occur if migration mortality is very high. Average relatedness increases with migration mortality $(C)$. Other details as in figure 3 .

own habitat patch network (Wahlberg et al. 1996). We analyze two patch networks of $M$. cinxia, of which the first is a relatively sparse one with 183 patches in a triangular area $23 \mathrm{~km}$ high and $29 \mathrm{~km}$ wide. The second network consists of 272 patches within an area $12 \mathrm{~km} \times 14 \mathrm{~km}$. As a consequence of the higher patch density in this second network, probability of surviving migration is $54 \%$, as compared to $44 \%$ in the sparse network. Average patch size is similar in both networks, 0.22 ha in the dense network and 0.23 ha in the sparse one.
Migration propensity evolves to a higher level in the sparse network $(\eta=0.118, \mathrm{SD}=0.006)$ than in the dense one $(\eta=0.104, \mathrm{SD}=0.006)$. The western half of the sparse network is so sparse that all local populations go extinct (fig. 8). Because of clustering of the patches, the rest of the network is fairly dense at the regional (withincluster) scale, and the probability of successful migration among the patches that were occupied after the transient was $51 \%$ in the sparse network and $55 \%$ in the dense one.
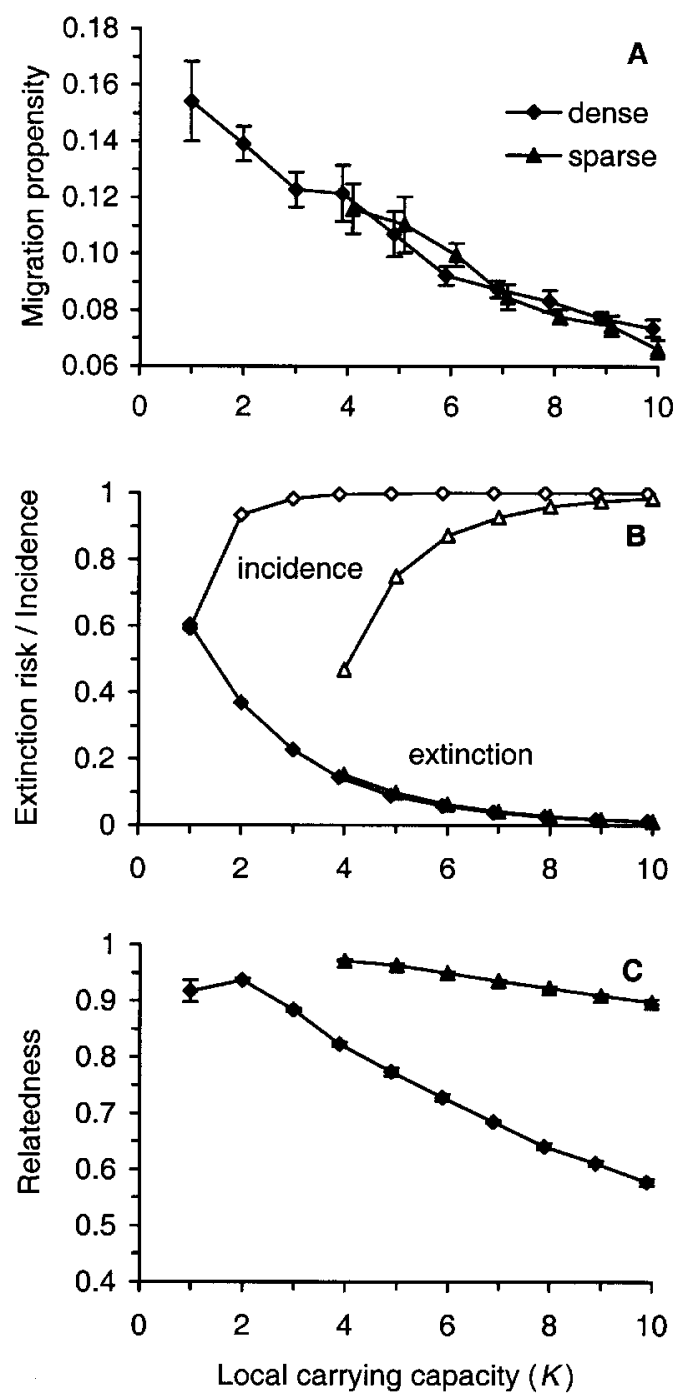

Figure 5: Influence of the carrying capacity on evolutionarily optimal migration propensity $(A)$. Emigration rate is typically higher in sparse (boxes) than in dense landscapes (diamonds). When the carrying capacity is very small, extinction risk is high and incidence is low $(B)$. Increasing the carrying capacity decreases relatedness $(C)$. Curves start from the lowest carrying capacity supporting a viable metapopulation. Other details as in figure 3. 

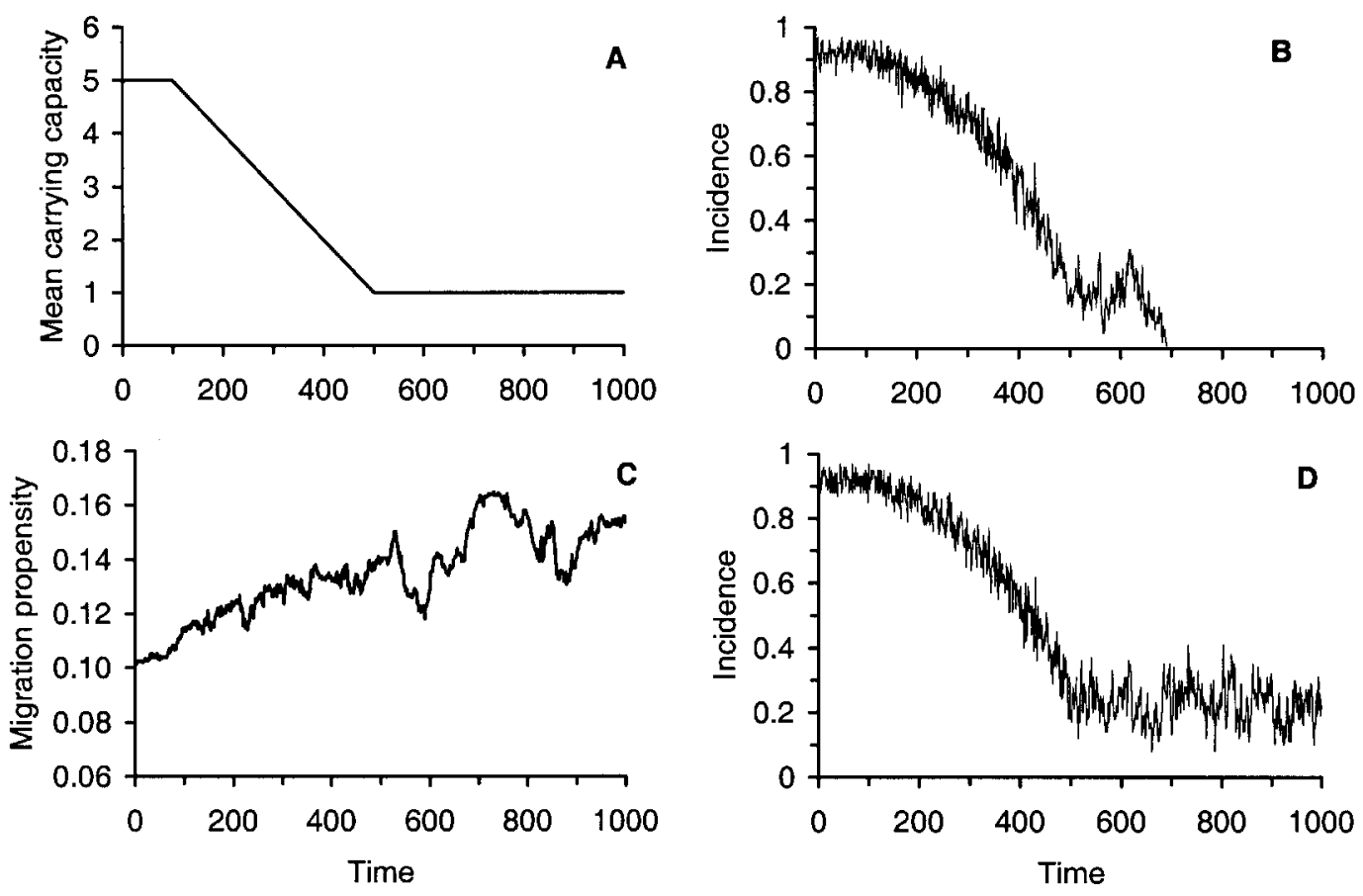

Figure 6: Evolutionary rescue in a metapopulation in a changing landscape. Habitat quality deteriorates between years 100 and 500 : the carrying capacity decreases from five to one $(A)$. If there is no evolution in the metapopulation, the habitat change leads to extinction $(B)$. However, decrease in the carrying capacity favors an increase of migration propensity $(C)$, and an evolving metapopulation can persist, albeit at a low level of incidence $(D)$. Both migration propensity and incidence fluctuate greatly in the deteriorated landscape because of very small local population sizes.

Clustering of patches in the sparse network allows regional differences in migration propensity to evolve (fig. $8 A$ ), whereas the dense network remains more homogeneous (fig. $8 D$ ). Differences in the average local extinction risk among patch clusters are large, and the migration propensity is positively correlated with extinction risk (fig. $8 E$ ). Combining data from the two Melitaea species (figs. $7 D, 8 E)$, there is a significant positive correlation $(r=$ 0.506 ) between extinction risk and migration propensity. Within-patch relatedness is very high in both networks, $0.968(\mathrm{SD}=0.003)$ in the sparse one and $0.953(\mathrm{SD}=$ $0.002)$ in the dense one.

\section{Discussion}

The model described and analyzed in this article is an individual-based simulation model. Such models have well-known shortcomings, of which the most obvious one is lack of generality-no exhaustive model analysis is possible. This drawback must be balanced against potential advantages. Provided that sufficient empirical information is available to justify model assumptions and to estimate parameter values, a carefully constructed simulation model can be tailored for particular systems to investigate, in a realistic manner, biologically significant questions. We consider that this approach is warranted in this case. First, we can employ two well-parameterized submodels to construct a large part of this model and to obtain empirical estimates of the parameter values (summarized in table 1). Second, the existing extensive knowledge about butterfly metapopulations and their landscapes (Thomas and Hanski 1997) helps focus the investigation on questions that have a broad significance not only for butterflies but for a large number of other taxa with similarly structured metapopulations (Hanski 1998, 1999). And third but not least, the model can be used to make testable predictions.

The number of model parameters is relatively large (15; table 1), but most of them can be estimated rigorously with empirical data. Structural assumptions about adult movement behavior and extinction-colonization dynamics are also well supported by empirical data. We therefore consider that our results are fairly robust and representative of the types of systems exemplified by the butterfly metapopulations. For the well-studied Melitaea diamina metapopulation, the model predicted an optimal migration propensity $(\eta=0.104, \mathrm{SD}=0.005)$ that is not significantly different from the empirically estimated value (0.130, $95 \%$ confidence limits $=0.103$ and 0.176 ; table 

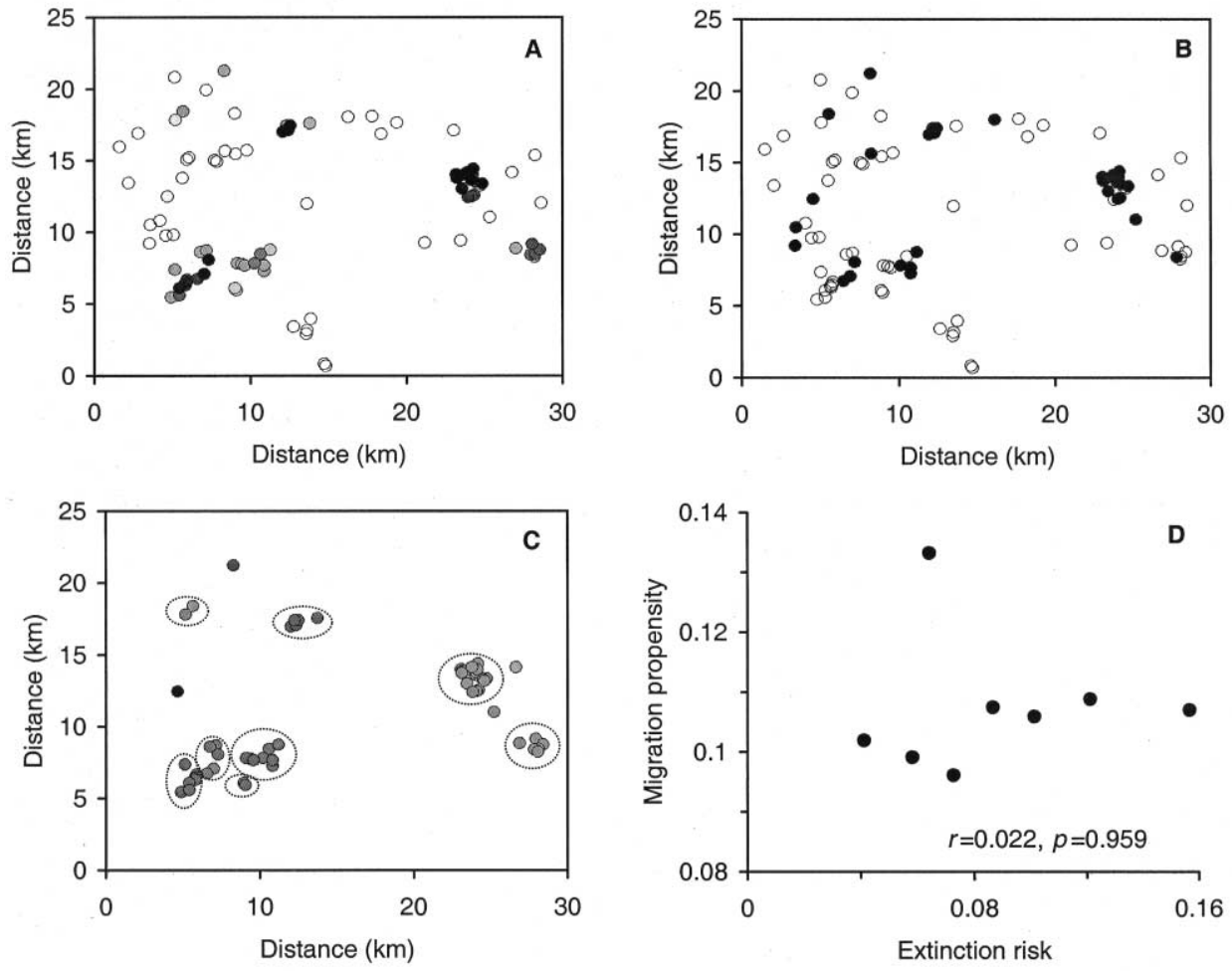

Figure 7: Predicted $(A)$ and the observed ( $B$; from Wahlberg et al. 1996) patterns of patch occupancy in the Melitaea diamina metapopulation. Shading of the dots indicates the incidence, from 0 (white) to 1 (black). $C$, Medium gray corresponds to $\eta=0.07$ and black to $\eta=0.14$. Regional differences in migration propensity emerge $(C)$. Average migration propensity within a patch cluster (the clusters used in the analysis are encircled in $C$ ) is not correlated with average extinction risk in the cluster $(D)$. In calculating within-cluster averages, patches were weighted with their carrying capacity. Delineation of clusters is based on the matrix $\psi$ (eq. [4]); isolated single patches or clusters with very low incidence are ignored. Ten replicate simulations were used.

1). Though this is not a critical test of the model structure nor of the optimality of the observed migration rate, the match between the observed and predicted values is encouraging.

Another shortcoming of individual-based models is that it is often difficult to know why a certain result is obtained. Of the processes that potentially influence the evolution of migration rate (Johnson and Gaines 1990; Olivieri and Gouyon 1997), some are included in our model whereas others are not. The key processes explicitly included are spatial and temporal variation in population density, including the extinction and reestablishment of local populations, resource and kin competition, and the direct cost of migration (elevated mortality during migration). Factors not included are inbreeding avoidance and everything else related to sexual reproduction, as well as any more complex behaviors leading to conditional migration, for instance, density-dependent emigration and immigration. Though these latter processes have some significance in many species, including the checkerspot butterflies studied here (Kuussaari et al. 1996, 1998; Saccheri et al. 1998), we consider that spatiotemporal variation in fitness as a result of stochastic population dynamics, mortality during migration, and kin competition are the primary determinants of the evolution of migration rate in many insect metapopulations, and hence this model represents a realistic baseline model.

Many of our evolutionary results can be explained both by selection associated with extinction-colonization dynamics and by kin selection arising from avoidance of kin competition. For example, a negative relationship between carrying capacity and migration rate can be explained both by changes in kin competition (Frank 1986; Taylor 1988; Gandon 1999; Gandon and Michalakis 1999) and by changes in extinction risk. However, in some cases, these two selective forces have opposing directions, allowing an assessment of which one is stronger in that particular case. Increasing mortality cost of migration up to moderate levels apparently selected for decreased migration rate, despite increasing relatedness (figs. 3, 4). Thus, selection 
Table 3: Consequences of habitat change in the metapopulation of Melitaea diamina

\begin{tabular}{lllll}
\hline & \multicolumn{3}{c}{ Occupancy (SD) } \\
\cline { 2 - 4 } Scenario & $\eta=.130$ & $\eta=.104$ & “Optimal” $\eta$ & $\eta($ SD) \\
\hline No habitat change & $.36(.04)$ & & $.36(.03)$ & $.104(.005)$ \\
20 patches lost & $.24(.04)$ & $.24(.05)$ & $.22(.04)$ & $.096(.007)$ \\
40 patches lost & $.15(.03)$ & $.16(.04)$ & $.14(.04)$ & $.098(.010)$ \\
20\% of patch area lost & $.20(.04)$ & $.21(.04)$ & $.18(.03)$ & $.104(.013)$ \\
$50 \%$ of patch area lost & $.19(.03)$ & $.21(.03)$ & $.18(.03)$ & $.101(.005)$ \\
$50 \%$ of $K$ lost & $.097(.023)$ & $.093(.015)$ & $.086(.015)$ & $.120(.017)$ \\
\hline
\end{tabular}

Note: Consequences based on the assumption that migration propensity has either the empirically estimated value $(\eta=0.130)$ or the optimal value in the original landscape $(\eta=0.104)$ or that $\eta$ is evolving. Habitat was changed by eradicating 20 or 40 randomly selected patches, by decreasing the areas of randomly selected patches by $50 \%$ until the desired amount of patch area was lost, or by decreasing carrying capacities (but not areas) of randomly selected patches by $50 \%$ until the desired amount of $K$ was lost. All values are based on 10 replicates of 1,100/1,500 generations (fixed $\eta$ /evolving $\eta$ ) with transients of $100 / 500$ generations omitted.

driven by extinction-colonization dynamics was stronger than kin selection in the cases where the predictions were different. This result is not due to lack of kin selection in our model, as can be shown by the following example.

The operation of kin selection can be demonstrated by artificially decreasing average within-population relatedness of individuals in a manner that leaves extinctioncolonization dynamics untouched. To achieve this, we ran six replicate simulations of the dense landscape, with an additional procedure of randomly mixing all the adult butterflies among the populations immediately before the start of migration in summer, without changing local population sizes nor dynamics. Such mixing completely eliminated elevated within-population relatedness ( $r$ decreased from $0.775[\mathrm{SD}=0.006]$ to $0.0037[0.0004])$, and there was a correlated response in the evolutionary optimal migration propensity, from 0.105 (0.008) to $0.062(0.009)$. If mixing is performed for larval groups, changes in relatedness and in migration propensity are less marked (average values from six replicates are $0.556[0.001]$ and 0.086 [0.006], respectively; recall that relatedness was calculated for adult individuals).

The assumption of asexual reproduction casts some doubt on our results regarding kin selection. For example, the relatedness estimates have probably been inflated. However, checkerspot butterflies mate only once and generally before migration, and hence the relatedness within a larval group is likely to be high. Therefore, asexuality is unlikely to be a critical assumption in our application of the model.

Many previous studies of the evolution of migration rate in metapopulations have focused on the consequences of local extinction rate on the optimal migration propensity. For some time, the consensus has been that the optimal migration propensity increases with extinction rate (van Valen 1971; Comins et al. 1980; Levin et al. 1984). Recently, Ronce et al. (2000) and Parvinen et al. (2000) have challenged this conclusion and suggested that the relationship is nonmonotonic, with very high extinction rate leading to reduced migration propensity. Unlike the previous authors, Ronce et al. (2000) and Parvinen et al. (2000) have studied structured metapopulation models, with an explicit description of local dynamics. High extinction rate shifts the distribution of local population sizes toward smaller populations (Hanski and Gyllenberg 1993), in which resource competition is weak and contributes little to selection for emigration (Ronce et al. 2000). On the other hand, if a large fraction of patches remain uncolonized because of low numbers of potential immigrants, expected fitness of an emigrant may be increased. The latter effect can occur in the model of Parvinen et al. (2000) but not in the model of Ronce et al. (2000). Both models treat colonization as a deterministic process.

In our model, local extinction is a mechanistic consequence of habitat patch size and stochastic local dynamics, which we have parameterized using empirical data. The combined data for the two Melitaea species reveals a monotonically increasing relationship between migration propensity and extinction risk. The same relationship holds for Melitaea cinxia alone (fig. 8E) but not for M. diamina (fig. 7D). Lack of relationship between extinction risk and $\eta$ in $M$. diamina is probably due to rather small differences among the patch clusters, too small for a significant relationship to emerge. In contrast, there are large differences in the extinction risk between the clusters of patches in the metapopulations of M. cinxia (fig. 8E).

To facilitate further comparison with the models of Ronce et al. (2000) and Parvinen et al. (2000), we increased local stochasticity in our model using the hypothetical dense landscape. We first increased variance in overwintering survival and/or family size. The resulting increase in local extinction risk selected for a monotonic increase in migration rate. The increase in extinction risk introduced in this man- 

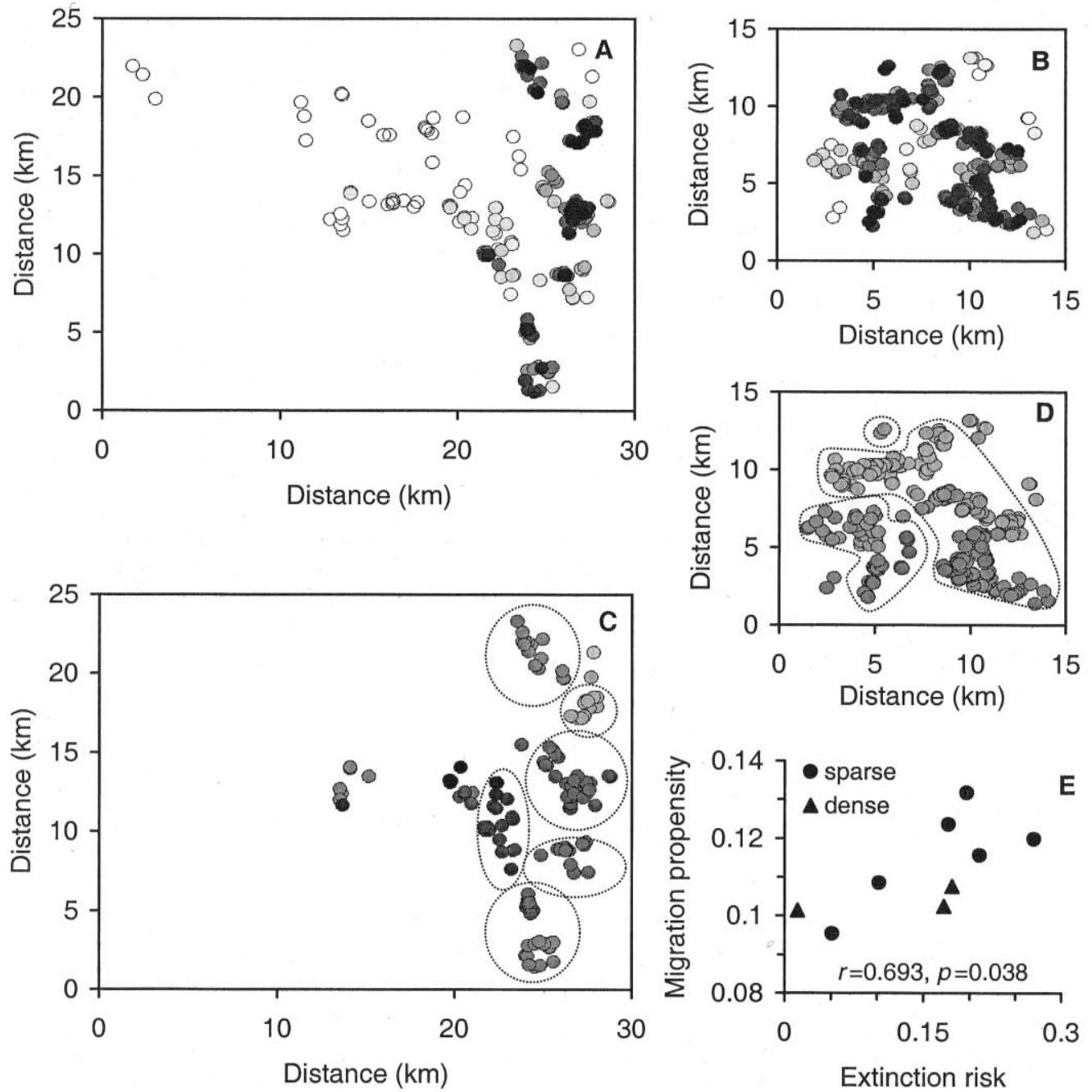

Figure 8: Predicted patch occupancy $(A, B)$ and migration propensity $(C, D)$ in two contrasting large habitat patch networks inhabited by Melitaea cinxia in the Åland Islands. The network in $A$ is sparse; only the eastern part of the network supports a viable metapopulation. Clustering of the patches allows some regional differences in migration propensity to evolve $(C)$. The other network $(B)$ is dense and less clustered. Migration propensity is fairly homogeneous over the network $(D)$. Within-cluster average migration propensity is correlated with average extinction risk $(E)$. Other details as in figure 7.

ner was not sufficient to cause a significant decrease in incidence nor in local population sizes after density dependence - the variances of survival during diapause and family size are bounded if the means are kept unchanged. However, a nonmonotonic relationship between extinction risk and migration propensity emerges in our model if we make a structural change by introducing exogenous extinctions (fig. 9 ). The eventual decrease in migration propensity occurs only close to the extinction threshold, when the annual probability of catastrophic extinction is exceedingly high, in the range $0.55-0.6$. Frequent local catastrophes leave population densities in the occupied patches unsaturated, which favors decreased emigration despite the large fraction of empty patches available for colonization. This effect becomes more pronounced for smaller larval group sizes. Note also that the predicted decrease in migration rate close to the extinction threshold is very slight, far from the dramatic decline in the model of Ronce et al. (2000). It remains to be seen whether or not the strong decline predicted by Ronce et al. (2000) can be observed in other realistic metapopulation models.

One biologically interesting question in the context of this model relates to the consequences of environmental change on migration rate. Several kinds of environmental 


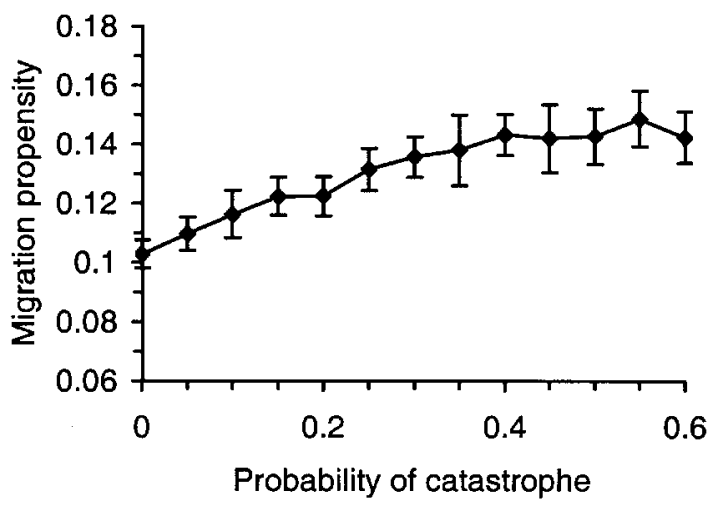

Figure 9: Influence of local catastrophes on the evolution of migration propensity. Catastrophes occur in summer after the migration period and destroy all the larvae in a habitat patch. Probability of catastrophe greater than $\sim 0.6$ leads to metapopulation extinction. The results are mean values of 10 (probability of catastrophe $<0.4$ ) or 20 replicates (otherwise) with standard deviations indicated by vertical bars. Other parameters as in table 1 .

changes can be considered with this model, including a change in the number and areas of the existing habitat patches, change in the quality of the patches (the carrying capacity, $K$ ), and change in the quality of the matrix habitat. Decreasing patch areas increase extinction rate (as $K$ decreases), whereas increasing patch isolation and reduced quality of the matrix habitat increase migration mortality. Note that our model assumes that individuals have the ability to distinguish between habitable patches and the uninhabitable matrix habitat, in contrast to models of diffusive dispersal analyzed by Travis and Dytham (1998, 1999).

We find it helpful to consider selection on migration propensity at two levels, at the level of local populations and at the level of the entire metapopulation, where the entities are local populations (though one can interpret all selection pressures from the perspective of an individual only). At the local level, increased migration is selected for by resource and kin competition and by reduced migration mortality. At the metapopulation level, finite lifetime of local populations guarantees the presence of empty patches that can only be colonized by migrating individuals. Selection for increased migration rate at the metapopulation level is limited by population turnover. The opposing selection pressures can lead to a nonmonotonic change in the optimal migration rate with environmental deterioration. Initially, when the metapopulation occupies most of the habitat, selection for reduced migration rate as a result of increasing migration mortality dominates, but eventually, when the landscape has deteriorated to a point where many patches remain empty, migration rate tends to increase. The nonmonotonic change has been predicted previously by Comins et al. (1980). Gandon and Michalakis (1999) demonstrate a similar effect based on competition among kin in a spatially implicit model. In our analyses, when the effects of kin competition and spatiotemporal variation in fitness could be disentangled, the latter appeared to be stronger.

Our results demonstrate how, in principle, an evolutionary change in migration rate can rescue a metapopulation from extinction in a deteriorating landscape (fig. 6). Whether or not evolutionary rescue is really important depends on the rate of environmental change in relation to the adaptive potential in the species (Lynch and Lande 1993; Gomulkiewicz and Holt 1995). The adaptive potential depends on population size and on genetic variance in the adaptive trait. In our model, the landscape structure largely determines the spatially structured population size, while the genetic variance is a function of the mutation rate and the genetic architecture of the trait. Unfortunately, as the mutation rate and genetic architecture are unknown, we cannot quantitatively assess the potential for evolutionary rescue. Our results lend limited support to both viewpoints-that evolutionary rescue is, and is not, of practical significance.

The good news for evolutionary rescue include the observation that, according to this model, considerable variation in migration propensity can be maintained in metapopulations as a result of weak phenotypic selection close to the evolutionary optimum (fig. 2). Furthermore, predictions for real butterfly metapopulations demonstrate considerable variation in the optimal migration propensity in different parts of single-patch networks, further adding to the variance in the trait value at the metapopulation level and demonstrating the potential for evolution in response to habitat change. Travis and Dytham (1999), analyzing another individual-based simulation model, also found regional adaptation in the migration rate, though in their lattice model with nearest-neighbor migration, differentiation occurred just between marginal and central "populations" living within a large expanse of suitable habitat (edge populations suffering from migration mortality). Some empirical studies have claimed to have found evidence for regional adaptation in migration rate in response to landscape structure (Thomas et al. 1998), but the evidence is not very conclusive. Note that this model can be used to make testable predictions about regional variation in migration rate in real landscapes (see figs. 7, 8), and it may thereby play a useful role in further studies of regional adaptation in migration rate.

Turning to the bad news, the predictions summarized in table 3 for various scenarios of change in a real landscape in which up to half of the pooled habitat was lost should temper our expectations about the potential sig- 
nificance of evolutionary rescue. In these examples, migration propensity indeed increased in the scenario with the greatest habitat loss, but the increase in optimal migration propensity was only moderate (from $\eta=0.104$ to 0.12 ), and most importantly, the consequences for metapopulation size were minimal, with the fraction of occupied patches being 0.09-0.1 for the relevant range of migration propensities. Such a tiny difference is likely to be completely swamped by additional stochastic effects in real environments. This example suggests that evolutionary rescue may have significant metapopulation dynamic consequences only in special circumstances. It should also be remembered that evolutionary change can be detrimental for a population. This might happen if local selection gradient favors a decrease in migration rate to a low level that precludes long-term persistence, even though some higher migration rate would allow persistence. However, we have not observed this phenomenon in our model.

Our spatially realistic individual-based metapopulation model complements existing simpler and more general models in two respects. First, it yields predictions that are directly applicable to real metapopulations. Some of these predictions can be tested, whereas others provide insight to management and conservation of real metapopulations. Second, this model helps evaluate the robustness of the predictions of simpler models, which necessarily make many unrealistic assumptions. It is encouraging that our results are consistent with the conclusions based on the earlier models, suggesting that many gross simplifications of analytical models do not matter that much. At the same time, we have been able to compare the relative contribution of particular processes to the evolution of migration rate, such as kin competition and spatiotemporal variation in fitness. In the kind of metapopulations considered here, our results indicate that spatiotemporal variation in fitness is more important than kin selection.

\section{Acknowledgments}

We thank N. Behera, M. Fred, S. Gandon, H. Kokko, A. Moilanen, M. Nieminen, P. Pamilo, N. Wahlberg, two anonymous referees, and the associate editor for very helpful advice, comments, and discussions and the Academy of Finland for financial support (project 45928 for M.H. and 44887 for I.H., Finnish Centre of Excellence Programme, 2000-2005).

\section{Literature Cited}

Adler, F. R., and B. Nuernberger. 1994. Persistence in patchy irregular landscapes. Theoretical Population Biology 45:41-75.

Brown, E. S. 1951. The relation between migration rate and type of habitat in aquatic insects, with special reference to certain species of Corixidae. Proceedings of the Zoological Society of London 121:539-545.

Comins, H. N., W. D. Hamilton, and R. M. May. 1980. Evolutionarily stable dispersal strategies. Journal of Theoretical Biology 82:205-230.

Frank, S. A. 1986. Dispersal polymorphisms in subdivided populations. Journal of Theoretical Biology 122:303-309.

Gadgil, M. 1971. Dispersal: population consequences and evolution. Ecology 52:253-261.

Gandon, S. 1999. Kin competition, the cost of inbreeding and the evolution of dispersal. Journal of Theoretical Biology 200:345-364.

Gandon, S., and Y. Michalakis. 1999. Evolutionarily stable dispersal rate in a metapopulation with extinctions and kin competition. Journal of Theoretical Biology 199: 275-290.

- 2001. Multiple causes of the evolution of dispersal. In J. Clobert, E. Danchin, A. A. Dhont, and J. D. Nichols, eds. Dispersal. Oxford University Press, Oxford (in press).

Gomulkiewicz, R., and R. D. Holt. 1995. When does evolution by natural selection prevent extinction? Evolution 49:201-207.

Hanski, I. 1994. A practical model of metapopulation dynamics. Journal of Animal Ecology 63:151-162.

- 1998. Metapopulation dynamics. Nature (London) 396:41-49.

. 1999. Metapopulation ecology. Oxford University Press, Oxford.

Hanski, I., and M. Gyllenberg. 1993. Two general metapopulation models and the core-satellite hypothesis. American Naturalist 142:17-41.

Hanski, I., and O. Ovaskainen. 2000. The metapopulation capacity of a fragmented landscape. Nature (London) 404:755-758.

Hanski, I., and D. Y. Zhang. 1993. Migration, metapopulation dynamics and fugitive coexistence. Journal of Theoretical Biology 163:491-504.

Hanski, I., A. Moilanen, T. Pakkala, and M. Kuussaari. 1996. The quantitative incidence function model and persistence of an endangered butterfly metapopulation. Conservation Biology 10:578-590.

Hanski, I., J. Alho, and A. Moilanen. 2000. Estimating the parameters of survival and migration of individuals in metapopulations. Ecology 81:239-251.

Hesse, R., W. C. Allee, and K. P. Schmidt. 1951. Ecological animal geography. Chapman \& Hall, New York.

Johnson, M. L., and M. S. Gaines. 1990. Evolution of dispersal: theoretical models and empirical tests using birds and mammals. Annual Reviews of Ecology and Systematics 21:449-480.

Kuussaari, M. 1998. Biology of the Glanville fritillary but- 
terfly (Melitaea cinxia). Ph.D. diss. University of Helsinki.

Kuussaari, M., M. Nieminen, and I. Hanski. 1996. An experimental study of migration in the butterfly Melitaea cinxia. Journal of Animal Ecology 65:791-801.

Kuussaari, M., I. Saccheri, M. Camara, and I. Hanski. 1998. Allee effect and population dynamics in the Glanville fritillary butterfly. Oikos 82:384-392.

Lei, G., and I. Hanski. 1998. Spatial dynamics of two competing specialist parasitoids in a host metapopulation. Journal of Animal Ecology 67:422-433.

Leimar, O., and U. Norberg. 1997. Metapopulation extinction and genetic variation in dispersal-related traits. Oikos 80:448-458.

Levin, S. A., D. Cohen, and A. Hastings. 1984. Dispersal strategies in patchy environments. Theoretical Population Biology 26:165-191.

Lynch, M., and R. Lande. 1993. Evolution and extinction in response to environmental change. Pages 234-250 in P. M. Kareiva, J. G. Kingsolver, and R. B. Huey, eds. Biotic interactions and global change. Sinauer, Sunderland, Mass.

McPeek, M. A., and R. D. Holt. 1992. The evolution of dispersal in spatially and temporally varying environments. American Naturalist 140:1010-1027.

Moilanen, A., A. T. Smith, and I. Hanski. 1998. Long-term dynamics in a metapopulation of the American pika. American Naturalist 152:530-542.

Olivieri, I., and P.-H. Gouyon. 1997. Evolution of migration rate and other traits: the metapopulation effect. Pages 293-323 in I. A. Hanski and M. E. Gilpin, eds. Metapopulation biology: ecology, genetics, and evolution. Academic Press, San Diego, Calif.

Pamilo, P. 1984. Genotypic correlation and regression in social groups: multiple alleles, multiple loci and subdivided populations. Genetics 107:307-320.

Parvinen, K., U. Dieckmann, M. Gyllenberg, and J. A. J. Metz. 2000. Evolution of dispersal in metapopulations with local density dependence and demographic stochasticity. IR-00-035. International Institute for Applied Systems Analysis, Laxenburg.

Queller, D. C., and K. F. Goodnight. 1989. Estimating relatedness using genetic markers. Evolution 43:258-275.

Roff, D. A. 1990. The evolution of flightlessness in insects. Ecological Monographs 60:389-421.
1994. Habitat persistence and the evolution of wing dimorphism in insects. American Naturalist 144: 772-798.

Ronce, O., F. Perret, and I. Olivieri. 2000. Evolutionarily stable dispersal rates do not always increase with local extinction rates. American Naturalist 155:485-496.

Ronce, O., J. Clobert, I. Olivieri, and E. Danchin. 2001. Perspectives on the study of dispersal evolution. In J. Clobert, E. Danchin, A. A. Dhont, and J. D. Nichols, eds. Dispersal. Oxford University Press, Oxford (in press).

Saccheri, I., M. Kuussaari, M. Kankare, P. Vikman, W. Fortelius, and I. Hanski. 1998. Inbreeding and extinction in a butterfly metapopulation. Nature (London) 392:491-494.

Southwood, T. R. E. 1962. Migration of terrestrial arthropods in relation to habitat. Biological Reviews of the Cambridge Philosophical Society 37:171-214.

Taylor, P. D. 1988. An inclusive fitness model for dispersal of offspring. Journal of Theoretical Biology 130:363-378.

ter Braak, C. F., I. A. Hanski, and J. Verboom. 1998. The incidence function approach to modeling of metapopulation dynamics. Pages 167-188 in J. Bascompte and R. V. Solé, eds. Modeling spatiotemporal dynamics in ecology. Springer, New York.

Thomas, C. D., and I. Hanski. 1997. Butterfly metapopulations. Pages 359-386 in I. A. Hanski and M. E. Gilpin, eds. Metapopulation biology: ecology, genetics, and evolution. Academic Press, San Diego, Calif.

Thomas, C. D., J. K. Hill, and O. T. Lewis. 1998. Evolutionary consequences of habitat fragmentation in a localised butterfly. Journal of Animal Ecology 67:485-497.

Travis, J. M. J., and C. Dytham. 1998. The evolution of dispersal in a metapopulation: a spatially explicit, individual-based model. Proceedings of the Royal Society of London B, Biological Sciences 265:17-23.

. 1999. Habitat persistence, habitat availability and the evolution of dispersal. Proceedings of the Royal Society of London B, Biological Sciences 266:723-728.

van Valen, L. 1971. Group selection and the evolution of dispersal. Evolution 25:591-598.

Wahlberg, N., A. Moilanen, and I. Hanski. 1996. Predicting the occurrence of endangered species in fragmented landscapes. Science (Washington, D.C.) 273:1536-1538. 